\title{
Thick target inverse kinematics approach for neutron emission
}

\author{
V. Z. Goldberg $\odot,,^{1, *}$ E. M. Gazeeva $\odot,{ }^{2,3}$ M. S. Golovkov, ${ }^{2,3}$ A. A. Bezbakh $\odot,{ }^{2}$ D. K. Nauruzbayev $\odot,{ }^{4,5}$ \\ A. K. Nurmukhanbetova, ${ }^{6}$ Zh. Kurmanaliyev ${ }^{0},{ }^{2,7,8}$ A. Serikov, ${ }^{2,3}$ B. Zalewski, ${ }^{2,9}$ and G. V. Rogachev (1) ${ }^{1,10,11}$ \\ ${ }^{1}$ Cyclotron Institute, Texas A\&M University, College Station, Texas 77843-3366, USA \\ ${ }^{2}$ Joint Institute for Nuclear Research, Dubna 141980, Russia \\ ${ }^{3}$ Dubna State University, Dubna 141982, Russia \\ ${ }^{4}$ Nazarbayev University, Nur-Sultan 010000, Kazakhstan \\ ${ }^{5}$ Saint Petersburg State University, Saint Petersburg 199034, Russia \\ ${ }^{6}$ Energetic Cosmos Laboratory, Nazarbayev University, Nur-Sultan 010000, Kazakhstan \\ ${ }^{7}$ L. N. Gumilyov Eurasian National University, Nur-Sultan 010000, Kazakhstan \\ ${ }^{8}$ Institute of Nuclear Physics, Almaty 050032, Kazakhstan \\ ${ }^{9}$ Heavy Ion Laboratory, PL-02-093 Warsaw, Poland \\ ${ }^{10}$ Department of Physics \& Astronomy, Texas A\&M University, College Station, Texas 77843-3366, USA \\ ${ }^{11}$ Nuclear Solutions Institute, Texas A\&M University, College Station, Texas 77843-3366, USA
}

(Received 17 April 2020; accepted 26 July 2020; published 12 August 2020)

\begin{abstract}
We applied the thick target inverse kinematics method for a study of resonances decaying through neutron emission. In this approach, the time of flight of the resonant neutrons is defined by the movement of the heavy ions of low-energy beams through an extended target. As a test, we used a well-studied, because of its role in the $s$ process in stars, ${ }^{13} \mathrm{C}(\alpha, n)$ reaction. The measurements demonstrated the high efficiency of the approach. The energy resolution for the ${ }^{13} \mathrm{C}(\alpha, n)$ excitation function was $\approx 60 \mathrm{keV}$, the largest contributions coming from the time structure of the beam and the thickness of the neutron detector. The measurements revealed a surprising disagreement with the $R$-matrix calculations based on the parameters of the recent analysis.
\end{abstract}

DOI: 10.1103/PhysRevResearch.2.032036

\section{INTRODUCTION}

About 30 years ago, one of us (V.Z.G.) proposed the thick target inverse kinematics (TTIK) method to search for $\alpha$-cluster states as scattering resonances in the interactions of heavy ions with ${ }^{4} \mathrm{He}$ [1]. In the TTIK technique, a beam of heavy ions is slowed in an extended helium gas target. The light recoils, $\alpha$ particles, are detected from a scattering event. These recoils emerge from the beam ions-helium interaction and hit a Si detector array located at the forward angles. The beam ions are stopped in the gas, while the $\alpha$ particles, having smaller energy losses, are not. Thus, the measurement of $\alpha$ recoil energies provides for a continuous excitation function of elastic scattering. Due to inverse kinematics, $0^{\circ}$ measurements correspond to $180^{\circ}$ c.m., and it is at this angle that the best energy resolution can be achieved. The energy resolution of $25 \mathrm{keV}$ c.m. has been achieved in Ref. [2]. Differences between the TTIK method and the classical approach are notable. In the classical approach, using beams with welldefined energies and very thin targets, one can yield an energy resolution of less than $1 \mathrm{keV}$ [3]. However, the classical

\footnotetext{
*goldberg@comp.tamu.edu

Published by the American Physical Society under the terms of the Creative Commons Attribution 4.0 International license. Further distribution of this work must maintain attribution to the author(s) and the published article's title, journal citation, and DOI.
}

approach makes systematic fine steps commensurate with the energy resolution infeasible. While the TTIK approach cannot compete with the classical approach in terms of energy resolution, it can provide systematic excitation functions, which are impractical to obtain in the standard approach. Initially, the TTIK approach has been proposed in experiments with stable beams, however, its greatest potential is for investigations of resonance scattering induced by rare, exotic beams [4].

The difference in the specific energy loss of a heavyion beam and the recoils is a very important issue for the implementation of the TTIK approach. The larger the difference, the better the resolution potentially achievable for the measurement of the excitation function. As neutrons have no direct ionization losses, the application of the TTIK method to cases with neutrons in a final state is tantalizing.

The present work applies the TTIK method to measurements of the excitation function of the ${ }^{13} \mathrm{C}(\alpha, n)$ reaction. We used the time-of-flight (TOF) technique (neutron detector time relative to the rf of the beam) for neutron energy measurements. The choice of the reaction was based on the fact that the excitation functions for the ${ }^{13} \mathrm{C}(\alpha, n)$ reaction have been studied at a broad range of $\alpha$-particle energies and because of the importance of this reaction for the nucleosynthetic $s$ process [5]. Additionally, our results can be compared to a very comprehensive and recent analysis of the data relevant to this reaction made by the University of Notre Dame group [6]; this reference also contains a comprehensive review of previous studies. 


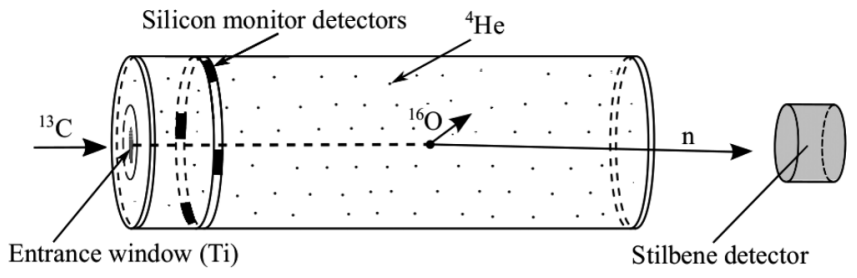

FIG. 1. Experimental layout.

\section{EXPERIMENT}

The experiment was performed at the DC-60 heavy-ion cyclotron in Nur-Sultan (Kazakhstan) using beam energies of 13.0 and $14.3 \mathrm{MeV}$. The cyclotron parameters were tuned to provide a beam bunch with optimal time resolution at the expense of beam intensity. The beam width was about $2 \mathrm{~ns}$ with $\approx 0.2 \%$ energy resolution at a beam intensity of a few $\mathrm{nA}$. The beam parameters were controlled by monitor detectors, described below. The experimental setup is presented in Fig. 1 . The cylindrically shaped scattering chamber $(15.5 \mathrm{~cm}$ diameter and $53.0 \mathrm{~cm}$ length) was made of stainless steel with a wall thickness of $0.25 \mathrm{~cm}$ and filled with helium of $99.99 \%$ purity.

The ${ }^{13} \mathrm{C}$ beam from the cyclotron entered the scattering chamber through a thin entrance window made of $1.9-\mu \mathrm{m} \mathrm{Ti}$ foil. Four monitor silicon detectors were mounted on a ringshaped holder inside the chamber in order to detect the ions elastically scattered from the entrance window. The energy of the beam decreases as it proceeds through the gas. While in most TTIK applications the unreacted beam is stopped in the gas, in this case, to increase the travel time of heavy ions between the resonances we used a lower pressure than needed to range out the beam. At a pressure of 25 Torr, the beam ions deposit about $25 \%$ of their energy in the gas and stop in a back flange of the scattering chamber. If the ${ }^{13} \mathrm{C}(\alpha, n)$ reaction occurs, a neutron flying forward near $0^{\circ}$ will pass through the solid angle subtended by a neutron detector $36 \mathrm{~cm}$ downstream, behind the back flange.

The neutron detector was a stilbene crystal of cylindrical shape ( $8 \mathrm{~cm}$ diameter, $5 \mathrm{~cm}$ thickness) optically coupled with a Photonis XP 4312/B photomultiplier. Stilbene has an excellent $n-\gamma$ separation ability which is exploited using standard pulse-shape discrimination (PSD) techniques. The amplitude calibration was made with ${ }^{60} \mathrm{Co}$ and ${ }^{137} \mathrm{Cs} \gamma$ sources. The time resolution for amplitudes exceeding $0.5 \mathrm{MeV}$ (equivalent electron energy) was better than $0.5 \mathrm{~ns}$ [full width at half maximum (FWHM)]. The detector efficiency was calculated using the data of Cherubini et al. [7]. Figure 2 demonstrates the quality of PSD made with the four-channel pulse-shape discriminator (MPD-4) module (Mesytec [8]), where the horizontal axis shows the amplitude in the recoil proton energy scale. Conversion from the electron to recoil proton scale was based on the data of Ref. [9]. We have made corrections for the detector size and for the threshold, which was $1.5 \mathrm{MeV}$ in the proton energy scale. Following Ref. [9], the amplitude of $0.5 \mathrm{MeV}$ in the electron scale corresponds to about $\approx 2.5 \mathrm{MeV}$ in the proton scale.

A neutron detector spectrum is shown in Fig. 3. The horizontal axis shows the neutron registration time relative to the

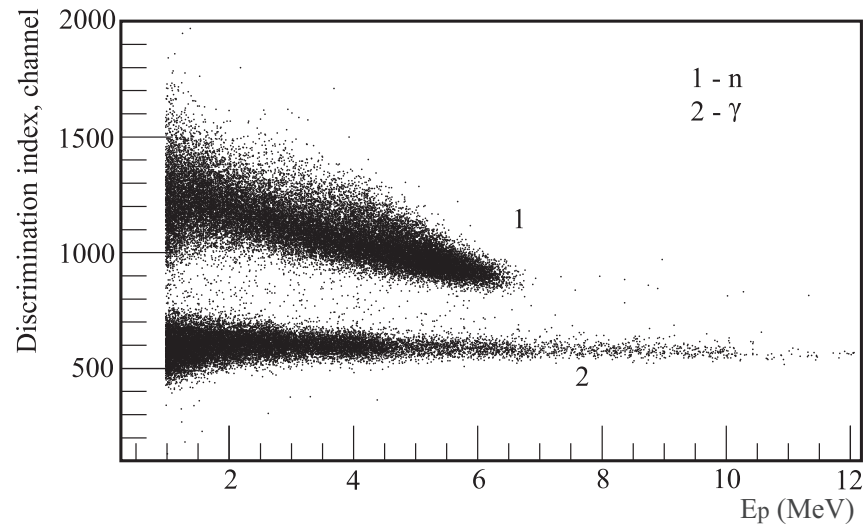

FIG. 2. $n-\gamma$ pulse-shape discrimination.

cyclotron $\mathrm{rf}$ and the vertical axis shows the amplitude in the recoil proton energy scale. The left (right) edge corresponds to the interaction of ${ }^{13} \mathrm{C}$ near the entrance window (downstream end of the chamber). The counts outside this interval cannot originate from the $\alpha-{ }^{13} \mathrm{C}$ interaction and provide a measure of the background. The background coming from window and back flange was measured without helium in the scattering chamber and was less than $1 \%$ of the neutrons from the ${ }^{13} \mathrm{C}(\alpha, n)$ reaction. Additional tests show that this background increases with energy of the beam as the penetrability of the Coulomb barrier. The neutron detector is placed rather close to the extended target. Therefore the neutron detection depends upon the solid angle of the reaction site in the target. It is rather unusual for the neutron TOF measurements, however,

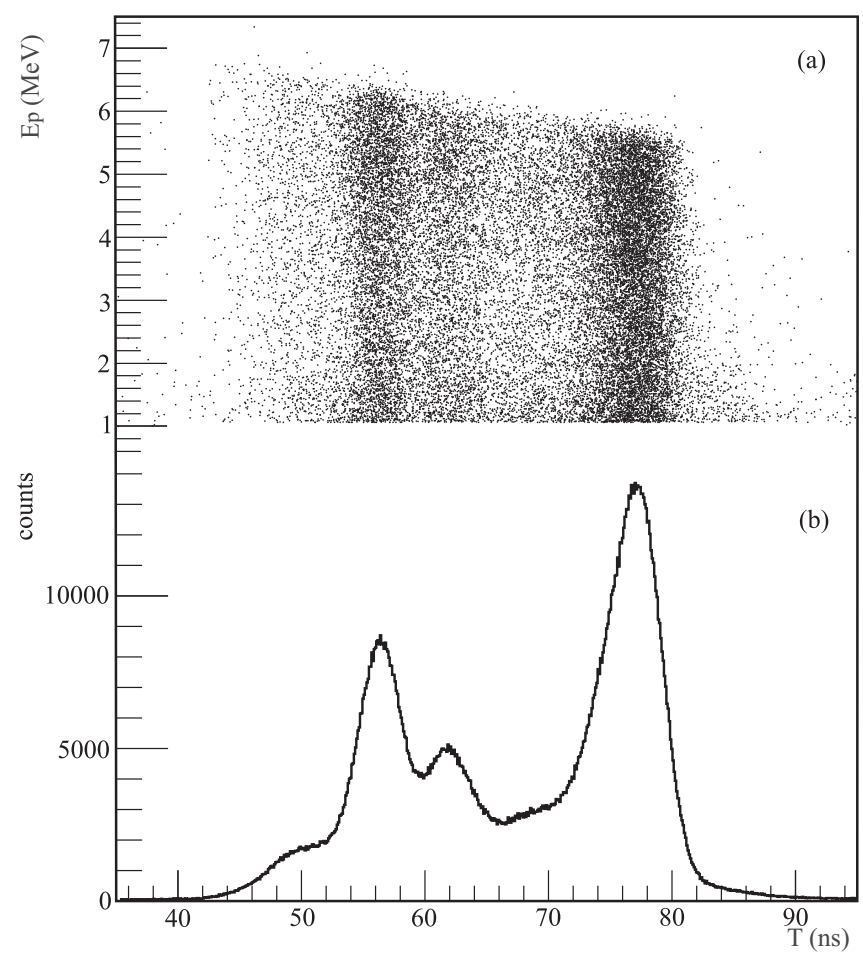

FIG. 3. (a) Two-dimensional $E_{p}$-TOF excitation function for the ${ }^{13} \mathrm{C}(\alpha, n)$ reaction and (b) projection of (a) spectrum on the $x$ axis (see the text for explanations). 


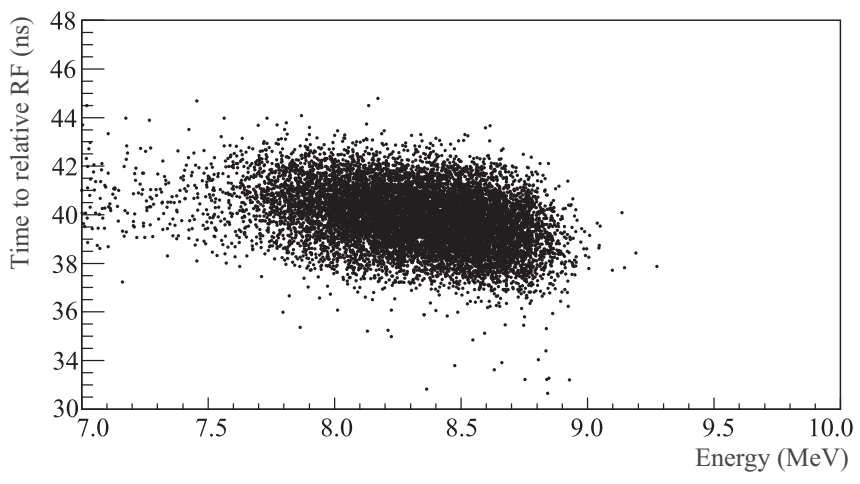

FIG. 4. Energy-time ( $E$-TOF) spectrum of the ions scattered from the entrance window.

it is common for the TTIK experiments [2]. The strong dependence upon a solid angle is partly compensated by a lower specific energy loss of the higher-energy ions near the entrance of the scattering chamber.

In order to consider the factors that influence the precision of the excitation function, a simulation of the experiment in the GEANT4 toolkit [10] was performed. We have obtained $63 \mathrm{keV}$ energy resolution for the ${ }^{13} \mathrm{C}(\alpha, n)$ excitation function based on the simulation, which is close to an estimation based on more transparent considerations.

However, before employing these simulations, it is instructive to obtain qualitative estimations of the time (energy) resolution using simple considerations. Figures 4 and 5 are provided to aid in this initial effort. One of these elementary considerations is the time dispersion of the beam ions as they reach the chamber. This dispersion is due to the initial energy spread of ions in the beam bunch and the flight path of $10 \mathrm{~m}$ between extraction from the cyclotron and the scattering chamber. Figure 4 shows the energy-time $(E-\mathrm{TOF})$ spectrum of the ions scattered from the entrance window observed in a monitor detector. It is seen in Fig. 4 that high-energy ions reach the detector earlier than the low-energy ions. The time difference of approximately $2 \mathrm{~ns}$ is in agreement with a conventional cyclotron energy resolution $( \pm 0.25 \%)$ and the 10 -m flight path. The detection angle of the monitor detectors

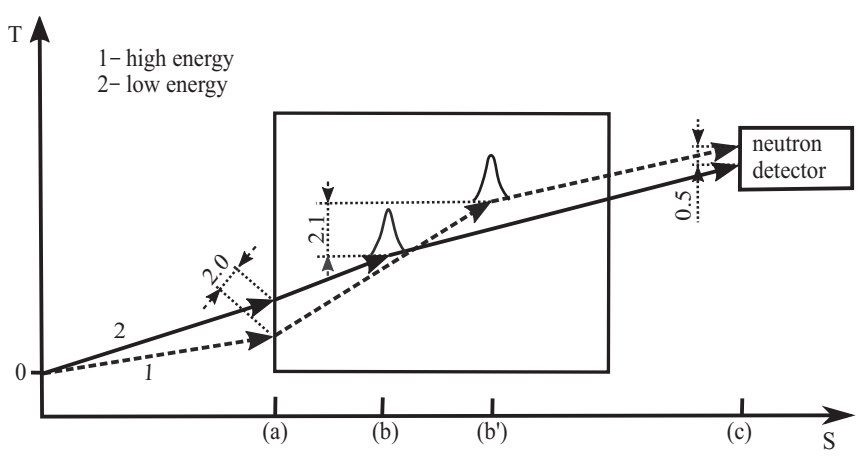

FIG. 5. Diagram of the development of time spread of neutrons in nanoseconds: (a) 1 (2) high- (low-) energy ions in the bunch; 1 is ahead of 2 at the entrance to the scattering chamber; (b) resonance place for 2; $\left(b^{\prime}\right)$ resonance place for 1 ; (c) 2 is ahead of 1 in the neutron detector (see the text for additional explanations).

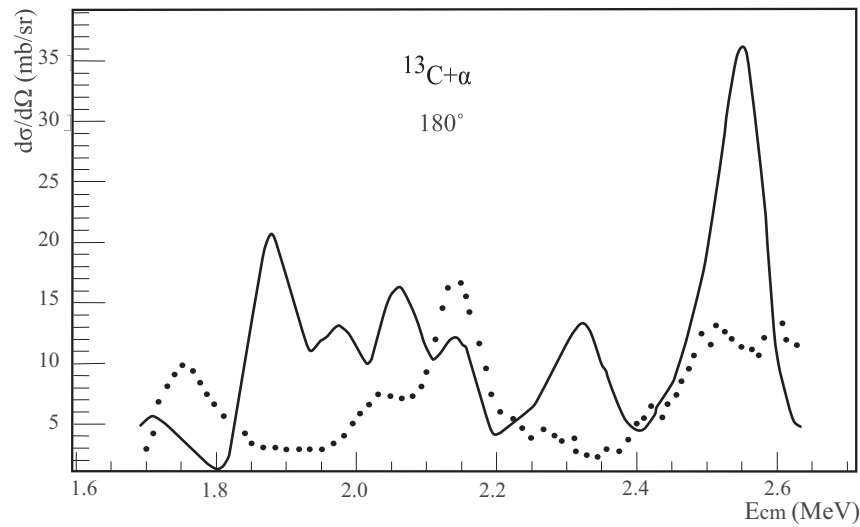

FIG. 6. The excitation function for the ${ }^{13} \mathrm{C}(\alpha, n)$ reaction at $180^{\circ}$. The bold curve is $R$-matrix calculations with the parameters of Ref. [6].

was $42^{\circ}$ with respect to the beam axis, which led to significant broadening (to $650 \mathrm{keV}$ ) of the peak shown in Fig. 4.

The derived energy spread of the beam after passing through the window foil is $450 \mathrm{keV}$. The main contribution to this spread is the nonuniformity of the thickness of the entrance window. This is estimated to be $9 \%$. These results are in agreement with previous observations of energy loss of different heavy ions in this foil [2].

For reference, and to revert to time, beam ions lose approximately $0.45 \mathrm{MeV}$ in $5.5 \mathrm{~cm}$ of gas and they need about $4.1 \mathrm{~ns}$ to travel this $5.5 \mathrm{~cm}$ distance. The low-energy ions of the beam reach resonance energy earlier, while the high-energy ions need to travel a longer distance (about $5.5 \mathrm{~cm}$ ) and deposit an extra $0.45 \mathrm{MeV}$ to reach the resonance energy. Therefore, the neutrons from the decay of a hypothetical narrow resonance in the ${ }^{13} \mathrm{C}(\alpha, n)$ reaction will originate with a time spread of $\approx(4.1-2.0)$ ns, with the low-energy part of the beam reaching the resonance earlier. Finally, the neutrons from the reaction induced by the lowest-energy part of the beam energy distribution should travel $5.5 \mathrm{~cm}$ more than those from the high-energy part to the detector at $0^{\circ}$. This difference amounts to about $1.6 \mathrm{~ns}$. (The neutron velocities are 2.5 higher than these of the heavy ions.) Summing up these effects: The faster ions from the machine get to the chamber sooner (about $2 \mathrm{~ns}$ ), but they have to travel farther (taking about $4.1 \mathrm{~ns}$ more), partially compensated by the reduced neutron flight time to the $0^{\circ}$ detector (about $1.6 \mathrm{~ns}$ ). The largely compensating effects lead to only an expected $0.5 \mathrm{~ns}$ time spread in the time of flight (TOF) of detected neutrons from the extreme ends of the initial energy distribution. To evaluate the overall time resolution, we should also take into account the original width of the beam bunch $(\approx 2 \mathrm{~ns})$, and the neutron detector resolution of $\approx 1.6$ ns (mainly from the finite thickness of the neutron detector). Considering all three as independent factors yields $2.5 \mathrm{~ns}$ (FWHM). This corresponds to a c.m. energy resolution of $\approx 60 \mathrm{keV}$.

Now, let us consider the excitation function obtained by our approach. Figure 6 demonstrates a combined spectrum using both the $13.0-$ and $14.3-\mathrm{MeV}{ }^{13} \mathrm{C}$ measurements. The overlapping region in these measurements was $\approx 2.73-2.87 \mathrm{MeV}$ c.m. The time-of-flight spectrum (Fig. 3) transformation to 

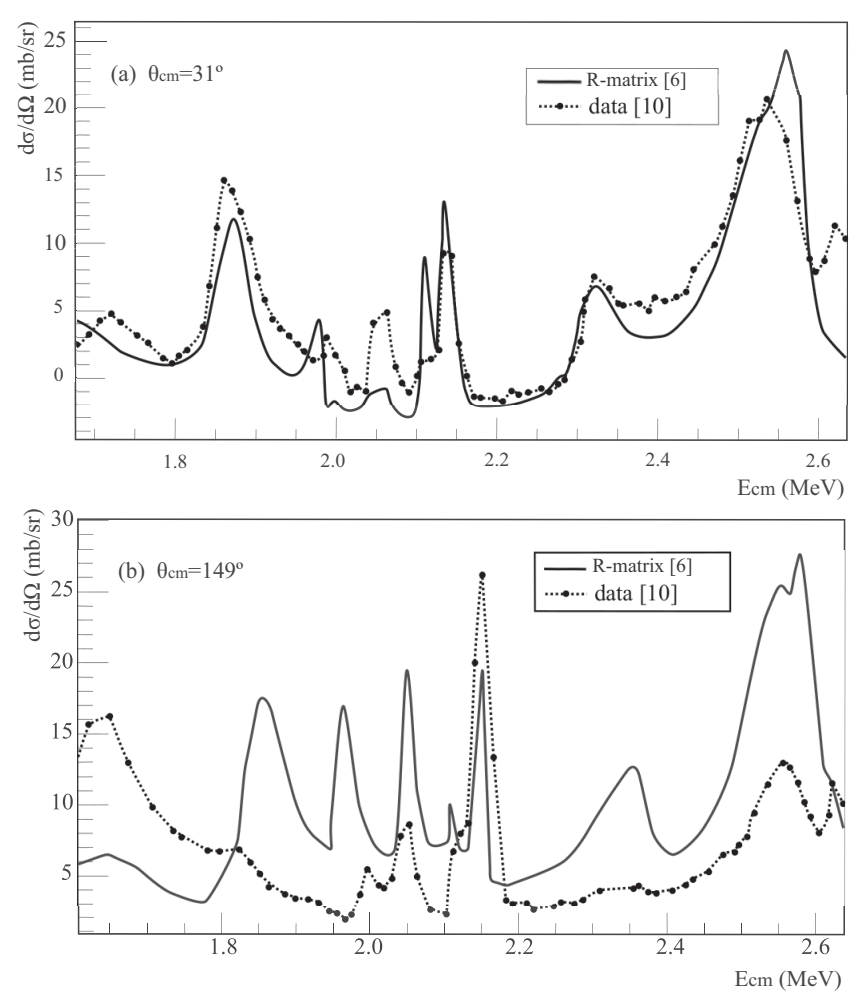

FIG. 7. The excitation function for the ${ }^{13} \mathrm{C}(\alpha, n)$ reaction at $31^{\circ}$ and $149^{\circ}$ from Ref. [12]. The bold curves are $R$-matrix calculations with the parameters of Ref. [6] (see the text for explanations).

the excitation functions (Fig. 6) is made using the conventional TTIK procedure [11]. The energy resolution estimated by the FWHM resolution for the peak at $2.15 \mathrm{MeV}$ c.m. energy is $\approx 60 \mathrm{keV}$. The solid line in Fig. 6 shows $R$-matrix calculations with the parameters of Ref. [6]. The disagreement between these calculations and our measurements is evident. Finding no obvious mistakes in either our work or that of the comprehensive work of Ref. [6], we looked to older experimental data for additional insight. The very old work by Walton et al. [12] did, in fact, provide some insight. These old excitation functions for the ${ }^{13} \mathrm{C}(\alpha, n)$ reaction at symmetric forward and backward angles $\left(31^{\circ}\right.$ and $\left.149^{\circ}\right)$ together with $R$-matrix calculations with contemporary parameters [6] are shown in Fig. 7. Fair agreement is found at the forward angles [Fig. 7(a)] but not at backward angles where the character of the disagreement is similar to that shown in Fig. 6. In spite of the evident difference in energy resolution, many specific details agree in the spectra of Figs. 6 and 7(b). The excitation energies of the peaks agree rather well in both measurements (the uncertainties of our measurements are $\approx 25 \mathrm{keV}$ ): $2.035 \mathrm{MeV}$ (Fig. 6) and 2.050 MeV [Fig. 7(b)], 2.15 and $2.15 \mathrm{MeV} ; 2.53$ and $2.56 \mathrm{MeV} ; 2.62$ and $2.63 \mathrm{MeV}$.

\section{DISCUSSION}

The present work shows that the energy resolution in the measurements of the excitation function for the ${ }^{13} \mathrm{C}(\alpha, n)$ reaction by the TTIK approach depends mainly upon the time width of the beam bunch (at the chamber) and on the time resolution of the neutron detector. Both factors can be improved and we are planning on doing so. We believe an energy resolution of $\approx 30 \mathrm{keV}$ is achievable. However, it is the high efficiency of this approach that is most noteworthy. The counting statistics in the observed peaks were well over $10^{5}$ counts. These data resulted from only 30 -h measurement with a beam intensity of a few $n A$.

The present measurements, planned as a test, revealed an interesting result. Evidently, this result is related to the unusual geometry. Obtaining equivalent data with the conventional approach would require a neutron measurement at angles close to $180^{\circ}$, a difficult measurement. The physical basis of the observed discrepancy likely resides in an unaccounted interference of one or more broad (low-spin) level(s) of opposite parity. A manifestation of such levels, if they are broad, might be attributed to a background in an analysis of forward angle data.

If this is the case, the unaccounted levels might influence the calculations for the lower excitation energy region important for astrophysics. However, a comprehensive analysis, such as that in Ref. [6], is needed to make a reliable conclusion.

It is not easy to predict the eventual applications of the specific TTIK approach described in the present work. (This was also the case in the original TTIK work [1].) One might imagine that the TTIK technique described here could be used to study $(\alpha, n)$ reactions on gas targets, many of which have not been thoroughly investigated. This is largely due to the experimental difficulties of working with gas targets in conventional geometry. The simplicity of the TTIK approach is attractive, and even more so when the reactions are induced by low-intensity secondary beams. In such cases, each beam particle would be tracked, removing a significant source of the ultimate c.m. energy uncertainty. As a final point, measurements of resonant gamma rays delayed by a movement of low-energy heavy ions in an extended target seem interesting. For such studies $\mathrm{LaBr}_{3}$ gamma detectors, with their excellent (respectable) time (energy) resolution, would be a good choice for these measurements. As a first test, gamma decays accompanying neutron (or proton) decays of the resonances to an excited state of a daughter nucleus might be selected because of the expected high yield.

\section{CONCLUSION}

We performed measurements of the excitation function for the ${ }^{13} \mathrm{C}(\alpha, n)$ reaction at $180^{\circ}$ c.m. using the TTIK method. The excitation function was obtained in the c.m. energy interval 1.7-2.6 MeV with an energy resolution of $\approx 60 \mathrm{keV}$ which is mainly defined by the width of the cyclotron beam burst and the neutron detector resolution. The obtained excitation function is in general agreement with an old measurement in the backward hemisphere [12]. However, the present results are in evident disagreement with a comprehensive fit [6] used to predict the ${ }^{13} \mathrm{C}(\alpha, n)$ reaction rate needed for astrophysics. We believe that the simplicity and high efficiency of the present approach will result in various applications. In particular, it can be used for the detection of $\gamma$ rays distributed in time according to the resonant interaction of low-energy heavy ions moving in an extended target. 


\section{ACKNOWLEDGMENTS}

The authors are indebted to Dr. S. Yennello for attention to the work and permanent support and to Dr. Lee Sobotka for reading the draft, for valuable comments, and the discussion. This work was supported in part by the Russian Science Foundation (Grant No. 17-12-01367). The authors also acknowledge financial support from the Nazarbayev University (Small Grant No. 090118FD5346), and the Ministry of Education and Science of the Republic of Kazakhstan (State-targeted
Program No. BR05236454 and Young Scientists' Research Grant No. AP08052268). This work was supported by the US Department of Energy, Office of Science, Office of Nuclear Science (Award No. DE-FG02-93ER40773) and by the National Nuclear Security Administration through the Center for Excellence in Nuclear Training and University Based Research (CENTAUR) (Grant No. DE-NA0003841). G.V.R. also acknowledges the support of the Welch Foundation (Grant No. A-1853).
[1] K. P. Artemov, O. P. Belyanin, A. L. Vetoshkin, R. Wolski, M. S. Golovkov, V. Z. Goldberg, M. Madeja, V. V. Pankratov, I. N. Serikov, V. A. Timofeev, V. N. Shadrin, and J. Szmider, Effective Method of Study of $\alpha$-cluster states, Sov. J. Nucl. Phys. USSR 52, 408 (1990).

[2] A. K. Nurmukhanbetova, V. Z. Goldberg, D. K. Nauruzbayev, G. V. Rogachev, M. S. Golovkov, N. A. Mynbayev, S. Artemov, A. Karakhodjaev, K. Kuterbekov, A. Rakhymzhanov, Z. Berdibek, I. Ivanov, A. Tikhonov, V. I. Zherebchevsky, S. Y. Torilov, and R. E. Tribble, Implementation of TTIK method and time of flight for resonance reaction studies at heavy ion accelerator DC-60, Nucl. Instrum. Methods Phys. Res., Sect. A 847, 125 (2017).

[3] W. J. Thompson, J. F. Wilkerson, T. B. Clegg, J. M. Feagin, E. J. Ludwig, and E. Merzbacher, Atomic-Excitation Effects on Nuclear Reactions, Phys. Rev. Lett. 45, 703 (1980).

[4] V. Goldberg, B. Roeder, G. Rogachev, G. Chubarian, E. Johnson, C. Fu, A. Alharbi, M. Avila, A. Banu, M. McCleskey, J. Mitchell, E. Simmons, G. Tabacaru, L. Trache, and R. Tribble, First observation of ${ }^{14} \mathrm{~F}$, Phys. Lett. B 5, 307 (2010).

[5] R. Gallino, C. Arlandini, M. Busso, M. Lugaro, C. Travaglio, O. Straniero, A. Chieffi, and M. Limongi, Evolution and nucleosynthesis in low-mass asymptotic giant branch stars. II. Neutron capture and the $s$-process, Astrophys. J. 497, 388 (1998).
[6] M. Heil, R. Detwiler, R. E. Azuma, A. Couture, J. Daly, J. Gorres, F. Kappeler, R. Reifarth, P. Tischhauser, C. Ugalde, and M. Wiescher, The ${ }^{13} \mathrm{C}(\alpha, n)$ reaction and its role as a neutron source for the $s$ process, Phys. Rev. C 78, 025803 (2008).

[7] R. Cherubini, G. Moschini, R. Nino, R. Policroniades, and A. Varela, Absolute neutron detection efficiency calibration of a stilbene organic scintillator in the energy range 2 to $20 \mathrm{MeV}$ with the associated particle technique, Nucl. Instrum. Methods Phys. Res., Sect. A 269, 623 (1988).

[8] Mesytec GmBH, Werner von Braun Strasse 1, D-85640 Putzbrunn, Germany, http://www.mesytec.com/.

[9] X. Zhang, X. Yuan, X. Xie, Z. Chen, X. Peng, J. Chen, G. Zhang, X. Li, T. Fan, G. Zhong, L. Hu, and B. Wan, A compact stilbene crystal neutron spectrometer for EAST D-D plasma neutron diagnostics, Rev. Sci. Instrum. 84, 033506 (2013).

[10] S. Agostinelli et al., Geant4-a simulation toolkit, Nucl. Instrum. Methods Phys. Res., Sect. A 506, 250 (2003).

[11] G. V. Rogachev, E. D. Johnson, J. Mitchell, V. Z. Goldberg, K. W. Kemper, and I. Wiedenhoever, Resonance scattering and alpha-transfer reactions for nuclear astrophysics, in Fifth European Summer School on Experimental Nuclear Astrophysics, edited by C. Spitaleri, C. Rolfs, and R. G. Pizzone, AIP Conf. Proc. Vol. 1213 (AIP, Melville, NY, 2010), p. 137.

[12] R. B. Walton, J. D. Clement, and F. Boreli, Interaction of neutrons with oxygen and a study of the $\mathrm{C}^{13}(\alpha, n) \mathrm{O}^{16}$ reaction, Phys. Rev. 107, 1065 (1957). 